\title{
Atividade de fosfito de potássio na pré e pós-infecção de Phakopsora euvitis em folhas de videira
}

\section{Potassium phosphite pre- and post-infection activities against Phakopsora euvitis in grapevine leaves}

\author{
Cláudia Regina Scapin Buffara ${ }^{1 *}$; Francislene Angelotti²; Dauri José Tessmann³; \\ Carolina Domingues de Souza ${ }^{4}$; João Batista Vida ${ }^{3}$
}

\begin{abstract}
Resumo
A ferrugem da videira, causada pelo fungo Phakopsora euvitis, é uma das principais doenças foliares da videira no Brasil. Este trabalho avaliou a atividade de fosfito de potássio na pré e pós-infecção da ferrugem da videira, comparativamente aos fungicidas hidróxido de cobre e tebuconazol. Os experimentos foram realizados com plantas de videira 'Niágara Rosada' com seis folhas (Vitis labrusca) cultivadas em vasos, em câmara de crescimento. As plantas foram pulverizadas com fungicidas até o ponto de escorrimento aos dois, cinco ou oito dias antes (efeito protetivo) ou após (efeito curativo) a inoculação com uma suspensão de urediniósporos $\left(10^{5}\right.$ esporos por $\left.\mathrm{mL}\right)$ de $P$. euvitis. Para quantificar a doença, foi avaliado o número de pústulas por $\mathrm{cm}^{2} \mathrm{em}$ quatro regiões do limbo foliar, de quatro folhas por planta. Em tratamento pré-infecção, o fosfito de potássio reduziu a quantidade de pústulas em até $64 \%$, quando aplicado até cinco dias antes da inoculação em relação ao tebuconazol. Hidróxido de cobre reduziu a quantidade de pústulas em $99 \%$. Em aplicações pós-infecção, fosfito de potássio reduziu a quantidade de pústulas em até $58 \%$, quando aplicado até dois dias antes da inoculação comparado a testemunha. Hidróxido de cobre reduziu a quantidade de pústulas em $60 \%$. O tratamento pós-infecção com fosfito de potássio foi menos eficaz na redução da germinação de urediniósporos do que o tratamento com hidróxido de cobre.
\end{abstract}

Palavras-chave: Doenças fúngicas, controle, fungos, Vitis

\begin{abstract}
Grapevine rust is caused by the fungus Phakopsora euvitis. It is a major foliar disease of grapevines in Brazil. In this study, the pre- and post-infection activities of potassium phosphite were compared to the standard grapevine fungicides copper hydroxide and tebuconazole. Experiments were conducted on 'Niagara Rosada' grapevines (Vitis labrusca) that were grown in pots. The plants were sprayed at two, five or eight days before (with protectant) or after (with a curative spray) inoculation with a urediniospore suspension $\left(10^{5}\right.$ spores per $\left.\mathrm{mL}\right)$ of $P$. euvitis until the suspension was running off the leaves. The resulting disease was quantified by the number of pustules per $\mathrm{cm}^{2}$ in four regions of each leaf blade in four leaves per plant. The pre-infection potassium phosphite treatment reduced pustule development by $64 \%$ when applied five days prior to rust inoculation, whereas the copper hydroxide and tebuconazole reduced pustule development by $99 \%$ and $100 \%$, respectively. The potassium
\end{abstract}

\footnotetext{
${ }^{1}$ Eng $^{\text {a }}$ Agra ${ }^{\mathrm{a}}$, Dr ${ }^{\mathrm{a}}$ em Proteção de Planta, Fitopatologia, Depto. de Agronomia, Universidade Estadual de Maringá, UEM, Maringá, PR. E-mail: claudiascapin@hotmail.com

2 Enga Agra ${ }^{\mathrm{a}}$ Dra da Embrapa Semi-Árido, Petrolina, PE. E-mail: fran.angelotti@cpatsa.embrapa.br

${ }^{3}$ Eng $^{\text {os }}$ Agr ${ }^{\text {os }}$, Profs. Drs. do Dept ${ }^{\circ}$ de Agronomia, UEM, Maringá, PR. E-mail: djtessmann@uem.br; jbvida@uem.br

${ }^{4}$ Eng $^{\mathrm{O}} \mathrm{Agr}^{\mathrm{o}}$, Discente do Curso de Mestrado em Proteção de Plantas, Fitopatologia, UEM, Maringá, PR. E-mail: carolinadsouza@ hotmail.com

* Autor para correspondência
} 
phosphite post-infection treatment reduced pustule development by $58 \%$ when applied two days after rust inoculation. The tebuconazole and copper hydroxide reduced disease development by $100 \%$ and $60 \%$, respectively, following post-infection treatment. Potassium phosphite had a weaker post-infection activity against urediniospore germination than copper hydroxide.

Key words: Fungal diseases, control, fungi, Vitis

\section{Introdução}

A ferrugem, causada pelo fungo Phakopsora euvitis (ONO, 2000), foi constatada no Brasil em 2001, no Paraná e em São Paulo (TESSMANN et al., 2004), tendo-se tornado, desde então, uma das principais doenças foliares da videira (Vitis spp.). Os prejuízos causados pela doença foram visualizados nas áreas vitícolas do país localizadas em regiões tropicais ou subtropicais com inverno ameno. Em parreirais de 'Niágara Rosada' (V. labrusca L.) no Paraná, os danos da doença geralmente ocorrem em cultivos tardios, quando a maturação dos frutos se prolonga até os meses de janeiro e fevereiro, ou então na segunda safra, realizada no período de janeiro a maio. Nessas condições, na ausência de controle químico, a doença pode causar perda total da produção devido a não maturação dos frutos, decorrente do desfolhamento precoce das plantas (VIDA; TESSMANN, 2005).

A ferrugem frequentemente ocorre concomitante a outras doenças da videira, algumas inclusive com maior potencial de danos, como o míldio, causado pelo oomyceto Plasmopara vitícola (Berk. \& M.A. Curtis) Berl. \& de Toni, o oídio e a podridão da uva madura, causados pelos fungos Uncinula necator (Schweinf.) Burrill e Glomerella cingulata (Stonemam) Spauld \& Schrenk, respectivamente. Dessas, o míldio é a doença que tem maior potencial de danos e por isso os parreirais podem receber mais de uma aplicação por semana visando seu controle (TESSMANN et. al., 2007). Fosfitos e hidróxido de cobre estão entre os fungicidas mais utilizados no controle de míldio, principalmente em aplicações pré-infecção ou protetivas, podendo ocorrer mais de oito aplicações de cada um desses produtos num mesmo ciclo de produção. De modo que, informações adicionais sobre atividades pré e pós- infecção (denominada curativa), desses fungicidas são importantes para os programas de manejo integrado de doenças da videira.

Os fosfitos, embora sejam comercializados como fertilizantes foliares, têm, reconhecidamente, atividade fungicida contra míldio da videira (REUVENI, 1997; SÔNEGO; GARRIDO; CZERMAINSKI, 2005; PEREIRA et al., 2010; GENTA et al., 2010; GOMES et al., 2011) e outros oomycetos (REBOLLAR-ALVITER; MADDEN; ELLIS, 2007; SILVA et al., 2011). São produtos originados da neutralização do ácido fosforoso $\left(\mathrm{H}_{3} \mathrm{PO}_{3}\right)$ por uma base, principalmente o hidróxido de potássio, formando o fosfito de potássio. Os fosfitos apresentam modo de ação sistêmico, translocação apo-simplástica, baixa toxicidade, baixo custo e atuam diretamente sobre os fungos, ativando o mecanismo de defesa das plantas ou induzindo a produção de fitoalexinas (SMILLIE; GRANT; GUEST, 1989; REUVENI, 1997; SÔNEGO; GARRIDO; CZERMAINSKI，2005; NOJOSA; RESENDE; RESENDE, 2005). Assim, por essas características, os fosfitos são opções válidas para serem incluídos em programas de manejo integrado de doenças da videira. Porém, o uso de fosfito para o controle pré e pós-infeção da ferrugem da videira ainda não foi quantitativamente avaliado em condições controladas.

Os fungicidas comerciais registrados para o controle da ferrugem da videira no Brasil são formulações de tebuconazol e da mistura de epoxiconazol com metiram. O primeiro trata-se de um fungicida sistêmico do grupo triazol que é utilizado também no controle de oídio e podridão da uva madura. O segundo é uma mistura de fungicida sistêmico do grupo estrobilurinas (epoxiconazol) com fungicida protetivo do grupo ditiocarbamato 
(metiram), o qual, além das doenças mencionadas para o tebuconazol, também é utilizado no controle de míldio (MAPA, 2012).

O objetivo deste trabalho foi avaliar a atividade de fosfito de potássio na pré e pós-infecção da ferrugem da videira, comparativamente aos fungicidas padrões hidróxido de cobre e tebuconazol. As informações geradas serão úteis em programas de manejo integrado de doenças da videira, contribuindo assim para a otimização e redução do uso de fungicidas na vitivinicultura tropical.

\section{Material e Métodos}

As mudas de videira 'Niágara Rosada' utilizadas nos experimentos foram produzidas em vasos com capacidade de $2 \mathrm{~L}$ de substrato, por meio de propagação de estacas contendo cinco gemas. Foi aplicada calcianamida $5 \%$ às gemas para quebra de dormência e obtenção de uniformidade na brotação.

A multiplicação dos urediniósporos utilizados como inóculo foi realizada em folhas de mudas de videira 'Niágara Rosada', em câmara de crescimento. Mudas com seis folhas foram infectadas por meio de pulverização, com uma suspensão de urediniósporos na concentração de $10^{5}$ esporos por $\mathrm{mL}$ em solução de Tween-20 a 0,01\%. Após a inoculação, as mudas foram submetidas à temperatura de 23$25^{\circ} \mathrm{C}$, com 24 horas de molhamento foliar, em ausência de luz. Posteriormente, as plantas foram mantidas em câmara de crescimento à temperatura de $23-25^{\circ} \mathrm{C}$ e fotoperíodo de 12 horas, conforme metodologia realizada por Angelotti et al., 2008. Os urediniósporos foram coletados 10 dias após a infecção das plantas.

Os tratamentos utilizados comparados nos ensaios que avaliaram as atividades pré e pósinfecção dos fungicidas foram os seguintes: 1) testemunha sem fungicida; 2) fosfito de potássio $\left.\left(1,5 \mathrm{~mL} \mathrm{~L}^{-1}\right) ; 3\right)$ fosfito de potássio $\left(3,0 \mathrm{~mL} \mathrm{~L}^{-1}\right)$; 4) fosfito de potássio $\left.\left(4,5 \mathrm{~mL} \mathrm{~L}^{-1}\right) ; 5\right)$ hidróxido de cobre $\left.9,7 \mathrm{~g} \mathrm{~L}^{-1}\right)$; 6) tebuconazol $\left(2,0 \mathrm{~g} \mathrm{~L}^{-1}\right)$. As doses dos tratamentos 2 e 5 seguem a recomendação para o controle de míldio da videira e a dose do tratamento 6 é recomendada para controle de ferrugem da videira.

No experimento realizado para avaliar a atividade pré-infecção, os fungicidas foram aplicados aos dois, cinco e oito dias antes da inoculação de $P$. euvitis, e no experimento realizado para avaliar a atividade pós-infecção, os fungicidas foram aplicados dois, cinco e oito dias após a inoculação. Em ambos os casos o delineamento experimental foi inteiramente casualizado, em esquema de fatores de variação fatorial, com três repetições, cada unidade experimental sendo constituída por um vaso com uma planta. A inoculação do patógeno e as condições de incubação foram às mesmas descritas anteriormente. Para quantificar a doença, foi avaliado o número de pústulas por $\mathrm{cm}^{2} \mathrm{em}$ quatro regiões do limbo foliar, de quatro folhas por planta. A quantificação foi feita com lupa de aumento de 20 vezes, acoplada a uma câmera fotográfica digital. As imagens obtidas foram transferidas para um computador, onde foram visualizadas para a contagem das pústulas no programa Windows Photo $^{\circledR}$. Esses experimentos foram realizados duas vezes.

Para a avaliação do efeito dos tratamentos na viabilidade dos urediniósporos, expressa pela porcentagem de germinação in vitro, os esporos foram coletados de plantas dos tratamentos que receberam aplicação pós-infecção de fungicida aos oito dias após a inoculação. Os esporos foram coletados 14 dias após a inoculação, com o auxílio de um pincel, realizando uma raspagem nas folhas para a retirada dos urediniósporos. Foi preparada uma suspensão de urediniósporos em água estéril na concentração de $10^{5}$ esporos por $\mathrm{mL}$ de cada uma das três repetições de cada tratamento e uma alíquota de $100 \mathrm{~mL}$ desta suspensão foi espalhada sobre a superfície de uma placa de Petri, contendo meio de ágar-água $2 \%$. Após 24 horas avaliou-se o percentual de esporos germinados e não-germinados em microscópio óptico. Foram considerados germinados os esporos 
com tubo germinativo de comprimento igual ou maior ao comprimento do esporo. $\mathrm{O}$ delineamento experimental foi inteiramente casualizado, com três repetições, sendo cada repetição constituída de uma placa de Petri.

A análise de variância dos dados e as comparações de médias dos tratamentos foram realizadas com o programa SISVAR (FERREIRA, 2011).

\section{Resultados e Discussão}

$\mathrm{Na}$ avaliação da atividade pré-infecção dos fungicidas, verificou-se que o número de pústulas de ferrugem nas folhas das plantas tratadas com fosfito de potássio, hidróxido de cobre e tebuconazol foi significativamente menor do que nas folhas das plantas testemunhas $(P=0,05)$ (Tabela 1$)$. Os tratamentos com fosfito de potássio foram menos eficientes na redução da infecção de $P$. euvitis do que hidróxido de cobre e tebuconazol. Inclusive, não ocorreu esporulação nas plantas tratadas com tebuconazol. Verificou-se interação fatorial significativa entre os tratamentos e os momentos de aplicação desse experimento. A aplicação de fosfito de potássio proporcionou reduções de $25 \%$ a $64 \%$ da doença em relação à testemunha. A aplicação de fosfito de potássio aos oito dias antes da inoculação mostrou menor efeito residual do que a aplicação aos dois e cinco dias antes da inoculação.

Tabela 1. Atividade pré-infecção. Número médio de pústulas de ferrugem e porcentagem de redução da doença em folhas de plantas de videira tratadas com fungicidas aos dois, cinco ou oito dias anteriores à inoculação (DAI) com urediniósporos de Phakopsora euvitis.

\begin{tabular}{|c|c|c|c|c|c|c|}
\hline \multirow[b]{2}{*}{ Tratamento } & \multicolumn{2}{|c|}{$2 \mathrm{DAI}$} & \multicolumn{2}{|c|}{$5 \mathrm{DAI}$} & \multicolumn{2}{|c|}{$8 \mathrm{DAI}$} \\
\hline & $\begin{array}{c}\mathrm{N}^{\mathrm{o}} \text { de } \\
\text { pústulas } \\
\text { por } \mathrm{cm}^{2}\end{array}$ & $\begin{array}{c}\text { Redução } \\
\text { da doença } \\
(\%)\end{array}$ & $\begin{array}{c}\mathrm{N}^{\mathrm{o}} \mathrm{de} \\
\text { pústulas } \\
\text { por } \mathrm{cm}^{2}\end{array}$ & $\begin{array}{c}\text { Redução } \\
\text { da doença } \\
(\%)\end{array}$ & $\begin{array}{c}\mathrm{N}^{\mathrm{o}} \mathrm{de} \\
\text { pústulas } \\
\text { por } \mathrm{cm}^{2}\end{array}$ & $\begin{array}{c}\text { Redução } \\
\text { da doença } \\
(\%)\end{array}$ \\
\hline Testemunha & 794 aA & 0 & 839 aA & 0 & $844 \mathrm{aA}$ & 0 \\
\hline Fosfito de potássio $1,5 \mathrm{ml} \mathrm{L}^{-1}$ & $422 \mathrm{bA}$ & 47 & $305 \mathrm{bB}$ & 64 & $521 \mathrm{bA}$ & 38 \\
\hline Fosfito de potássio $3,0 \mathrm{ml} \mathrm{L}^{-1}$ & $423 \mathrm{bB}$ & 47 & $385 \mathrm{bB}$ & 54 & $626 \mathrm{bA}$ & 26 \\
\hline Fosfito de potássio $4,5 \mathrm{ml} \mathrm{L}^{-1}$ & $295 \mathrm{cB}$ & 63 & $391 \mathrm{bB}$ & 53 & $637 \mathrm{bA}$ & 25 \\
\hline Hidróxido de cobre $9,7 \mathrm{~g} \mathrm{~L}^{-1}$ & $4 \mathrm{dA}$ & 99 & $4 \mathrm{cA}$ & 99 & $2 \mathrm{cA}$ & 99 \\
\hline Tebuconazol 2,0 $\mathrm{g} \mathrm{L}^{-1}$ & $0 \mathrm{dA}$ & 100 & $0 \mathrm{cA}$ & 100 & $0 \mathrm{cA}$ & 100 \\
\hline
\end{tabular}

${ }^{1}$ Formulação $40 \% \mathrm{P}_{2} \mathrm{O}_{5}$ e $20 \% \mathrm{~K}_{2} \mathrm{O}$.

Médias seguidas por letras distintas, minúsculas na vertical e maiúsculas na horizontal, diferem entre si, ao nível de $5 \%$ de probabilidade, pelo teste de Scott Knott. CV $=10,7 \%$. Para a análise estatística os dados foram transformados para arc sen $\sqrt{ } \mathrm{x} / 100$.

Fonte: Elaboração dos autores.

$\mathrm{Na}$ avaliação da atividade pós-infecção dos fungicidas, verificou-se controle significativo dos tratamentos com fungicidas em relação à testemunha $(P=0,05)$ (Tabela 2$)$. Os tratamentos com fosfito de potássio foram menos eficientes na redução da infecção de $P$. euvitis do que o tratamento com tebuconazol. Também verificou-se a interação fatorial significativa entre os tratamentos e os momentos de aplicação. A aplicação de fosfito de potássio proporcionou reduções de $22 \%$ a $58 \%$ da doença em relação à testemunha. $(P=0,05)$ 
Tabela 2. Atividade pós-infecção. Número médio de pústulas de ferrugem em folhas de plantas de videira tratadas com fungicidas aos dois, cinco ou oito dias posteriores à inoculação (DPI) com urediniósporos de Phakopsora euvitis.

\begin{tabular}{|c|c|c|c|c|c|c|}
\hline \multirow[b]{2}{*}{ Tratamento } & \multicolumn{2}{|c|}{2 DAI } & \multicolumn{2}{|c|}{$5 \mathrm{DAI}$} & \multicolumn{2}{|c|}{$8 \mathrm{DAI}$} \\
\hline & $\begin{array}{c}\mathrm{N}^{\mathrm{o}} \text { de } \\
\text { pústulas } \\
\text { por } \mathrm{cm}^{2}\end{array}$ & $\begin{array}{c}\text { Redução } \\
\text { da doença } \\
(\%)\end{array}$ & $\begin{array}{c}\mathrm{N}^{\mathrm{o}} \mathrm{de} \\
\text { pústulas } \\
\text { por } \mathrm{cm}^{2}\end{array}$ & $\begin{array}{c}\text { Redução } \\
\text { da doença } \\
(\%)\end{array}$ & $\begin{array}{c}\mathrm{N}^{\mathrm{o}} \mathrm{de} \\
\text { pústulas } \\
\text { por } \mathrm{cm}^{2}\end{array}$ & $\begin{array}{c}\text { Redução } \\
\text { da doença } \\
(\%)\end{array}$ \\
\hline Testemunha & $860 \mathrm{eA}$ & 0 & $804 \mathrm{dA}$ & 0 & $810 \mathrm{dA}$ & 0 \\
\hline Fosfito de potássio ${ }^{1} 1,5 \mathrm{ml} \mathrm{L}^{-1}$ & $642 \mathrm{~dB}$ & 25 & $498 \mathrm{cA}$ & 38 & $628 \mathrm{cB}$ & 22 \\
\hline Fosfito de potássio $3,0 \mathrm{ml} \mathrm{L}^{-1}$ & $359 \mathrm{bA}$ & 58 & $558 \mathrm{cB}$ & 31 & $468 \mathrm{bB}$ & 42 \\
\hline Fosfito de potássio $4,5 \mathrm{ml} \mathrm{L}^{-1}$ & $391 \mathrm{bA}$ & 55 & $490 \mathrm{cA}$ & 39 & $486 \mathrm{bA}$ & 40 \\
\hline Hidróxido de cobre 9,7 g L-1 & $507 \mathrm{cB}$ & 41 & $319 \mathrm{bA}$ & 60 & $404 \mathrm{bA}$ & 50 \\
\hline Tebuconazol 2,0 $\mathrm{g} \mathrm{L}^{-1}$ & $0 \mathrm{aA}$ & 100 & $0 \mathrm{aA}$ & 100 & $0 \mathrm{aA}$ & 100 \\
\hline
\end{tabular}

${ }^{1}$ Formulação: $40 \% \mathrm{P}_{2} \mathrm{O}_{5}$ e $20 \% \mathrm{~K}_{2} \mathrm{O}$.

Médias seguidas por letras distintas, minúsculas na vertical e maiúsculas na horizontal, diferem entre si, ao nível de $5 \%$ de probabilidade, pelo teste de Scott Knott. $C V=10,3 \%$. Para a análise estatística os dados foram transformados para arc sen $\sqrt{ } \mathrm{x} / 100$.

Fonte: Elaboração dos autores.

Aplicações de hidróxido de cobre e de fosfito de potássio em pós-infecção de $P$. euvitis, aos oito dias após a inoculação, ou seja, em pústulas esporulantes, reduziu significativamente a germinação de urediniósporos em relação à testemunha sem fungicida $(P=0,05)$ (Tabela 3), porém hidróxido de cobre reduziu mais a germinação dos esporos do que fosfito de potássio.

Tabela 3. Porcentagem de germinação e germinação relativa de urediniósporos de Phakopsora euvitis coletados em folhas de plantas de videira tratadas com fungicidas aos oito dias posteriores à inoculação.

\begin{tabular}{|c|c|c|}
\hline Tratamento & $\begin{array}{c}\text { Germinação } \\
(\%)\end{array}$ & $\begin{array}{c}\text { Germinação relativa } \\
(\%)\end{array}$ \\
\hline Testemunha & $86 \mathrm{a}$ & 100 \\
\hline Fosfito de potássio ${ }^{1} 1,5 \mathrm{ml} \mathrm{L}^{-1}$ & $64 \mathrm{~b}$ & 74 \\
\hline Fosfito de potássio $3,0 \mathrm{ml} \mathrm{L}^{-1}$ & $61 \mathrm{~b}$ & 71 \\
\hline Fosfito de potássio $4,5 \mathrm{ml} \mathrm{L}^{-1}$ & $62 \mathrm{~b}$ & 72 \\
\hline Hidróxido de cobre $9,7 \mathrm{~g} \mathrm{~L}^{-1}$ & $13 \mathrm{c}$ & 15 \\
\hline
\end{tabular}

${ }^{1}$ Formulação: $40 \% \mathrm{P}_{2} \mathrm{O}_{5}$ e $20 \% \mathrm{~K}_{2} \mathrm{O}$.

Médias seguidas por letras distintas, minúsculas na vertical e maiúsculas na horizontal, diferem entre si, ao nível de $5 \%$ de probabilidade, pelo teste de Scott Knott. CV $=10,7 \%$. Para a análise estatística os dados foram transformados para arc sen $\sqrt{ } \mathrm{x} / 100$. Fonte: Elaboração dos autores.

Este estudo mostrou que o tratamento com fosfito de potássio reduz a colonização de $P$. euvitis em folhas de videira, tanto em aplicações pré-infecção ou protetivas, como em aplicações pós-infecção ou curativas. O estudo mostrou alta eficiência do fungicida tebuconazol no controle protetivo e curativo da ferrugem da videira, fato que já foi caracterizado em relação à ferrugem asiática da soja, causada por P. pachyrhizi H. Sydow \& Sydow (GODOY; CANTERI, 2004). Enquanto que o hidróxido de cobre ficou caracterizado como fungicida com boa atividade protetiva e atividade curativa limitada.

Há controvérsias na literatura quanto à eficiência de fosfitos no controle de ferrugens. Com relação 
à $P$. euvitis, Gomes et al. (2011) verificaram que a aplicação de fosfito de potássio não reduziu significativamente a incidência de ferrugem da videira nas folhas de plantas com infecção natural, em condições de campo. Porém, ressaltase que naquele estudo ocorreu baixo índice de incidência da doença nas plantas, com média de 4\% de incidência na testemunha, o que talvez não tenha permitido captar os efeitos dos tratamentos. Neves (2006) mostrou que a aplicação de fosfito de potássio reduziu a severidade da doença no início da epidemia, verificando que nos primeiros 30 dias após a aplicação, a severidade da doença foi de $5,06 \%$ nas parcelas tratadas com fosfito de potássio, $1,08 \%$ nas parcelas tratadas com tebuconazol e $15 \%$ nas parcelas testemunhas. Entretanto, Meneghetti et al. (2010) verificaram em estudo realizado em casa de vegetação que o uso de fosfito não reduziu a colonização da ferrugem asiática da soja em plantas tratadas aos 3, 7 e 14 dias antes e após a inoculação. Porém, os autores não mencionaram qual foi o fosfito utilizado no estudo, ou seja, o produto da neutralização de $\mathrm{H}_{3} \mathrm{PO}_{3}$ por uma base, tornando assim difícil a comparação de resultados. Em um terceiro estudo, com a ferrugem da folha do trigo (Puccinia triticina Erikss) em condições de campo, Santos et al. (2011) verificaram que fosfito de potássio não teve efeito significativo na redução da severidade da doença, porém não informam se os tratamentos foram aplicados antes ou depois da infecção.

O presente estudo mostrou que aplicações de fosfito de potássio antes e após a inoculação de $P$. euvitis reduzem a colonização das folhas de videira pelo patógeno, muito embora com limitações. Os resultados também mostram que a aplicação de fosfito de potássio tanto em pré-infecção como em pós-infecção pode reduzir a doença em até aproximadamente $50 \%$ quando comparado ao tratamento com tebuconazol. Esses resultados não são desprezíveis considerando-se que normalmente são realizadas de 5 a 10 aplicações de fosfitos, geralmente com intervalos de uma semana, durante o ciclo de produção de videira visando o controle de míldio. Este estudo mostrou também que hidróxido de cobre, um fungicida também muito utilizado no controle de míldio, apresenta boa atividade pré-infecção, como fungicida protetivo e que sua atividade pós-infecção ou curativa equivale aproximadamente ao fosfito de potássio. Assim, espera-se que as informações geradas neste trabalho sejam úteis para o manejo integrado de doenças da videira.

\section{Agradecimentos}

À CAPES (Coordenação de Aperfeiçoamento de Pessoal de Nível Superior) pela concessão da bolsa e apoio financeiro no desenvolvimento deste trabalho.

\section{Referências}

ANGELOTTI, F.; SCAPIN, C. R.; TESSMANN, D. J.; VIDA, J. B.; VIEIRA, R. A.; DE SOUTO, E. E. R. Resistência de genótipos de videira à ferrugem. Pesquisa Agropecuária Brasileira, Brasília, v. 43, n. 9, p. 11291134, 2008.

FERREIRA, D. F. SISVAR: a computer statistical analysis system. Ciência e Agrotecnologia, Lavras, v. 35, n. 6, p. 1039-1042, 2011.

GENTA, W.; TESSMANN, D. J.; ROBERTO, S. R.; VIDA, J. B.; COLOMBO, L. A.; SCAPIN, C. R.; RICCE, W. S.; CLOVIS, L. R. Manejo de míldio no cultivo protegido de videira de mesa 'BRS Clara'. Pesquisa Agropecuária Brasileira, Brasília, v. 45, n. 12, p. 13881395, 2010.

GODOY, C. V.; CANTERI, M. G. Efeitos protetor, curativo e erradicante de fungicidas no controle da ferrugem asiática da soja por Phakopsora pachyrhizi, em casa de vegetação. Fitopatologia Brasileira, Brasília, v. 29, n. 1, p. 97-101, 2004.

GOMES, E. C. S.; LEITE, R. P.; SILVA, F. J. A.; CAVALCANTI, L. S.; NASCIMENTO, L. C.; SILVA, S. M. Manejo do míldio e ferrugem em videira com indutores de resistência: produtividade e qualidade póscolheita. Tropical Plant Pathology, Brasília, v. 36, n. 5, p. 332-335, 2011. 
MAPA - Ministério da Agricultura. Fungicidas comerciais registrados para o controle da ferrugem da videira no Brasil. Brasília, 2012. Disponível em: <http:// agrofit.agricultura.gov.br/agrofit_cons/principal_agrofit cons>. Acesso em: 10 abr. 2012.

MENEGHETTI, R. C.; BALARDIN, R. S.; CORTE, G. D.; FAVERA, D. D.; DEBONA, D. Avaliação da ativação de defesa em soja contra Phakopsora pachyrhizi em condições controladas. Ciência e Agrotecnologia, Lavras, v. 34, n. 4, p. 823-829, 2010.

NEVES, J. S. Influência da aplicação de fosfito de potássio na severidade da ferrugem asiática da soja. 2006. Dissertação (Mestrado em Agronomia) Universidade de Brasília, Faculdade de Agronomia e Veterinária, Brasília.

NOJOSA, G. B. de A.; RESENDE, M. L. V.; RESENDE, A. V. Uso de fosfitos e silicatos na indução de resistência. In: CAVALCANTI, L. S.; DI PIERO, R. M.; CIA, P.; PASCHOLATI, S. F.; RESENDE, M. L. V.; ROMEIRO, R. S. Indução de resistência em plantas a patógenos e insetos. Piracicaba: FEALQ, 2005. 263 p.

ONO, Y. Taxonomy of the Phakopsora ampelopsidis species complex on vitaceous hosts in Asia including a new species, Phakopsora euvitis. Mycologia, Lawrence, USA, v. 92, n. 1, p. 154-173, 2000.

PEREIRA, V. F.; RESENDE, M. L. V. de; MONTEIRO, A. C. A.; RIBEIRO JÚNIOR, P. M.; REGINA, M. A.; MEDEIROS, F. C. L. Produtos alternativos na proteção da videira contra o míldio. Pesquisa Agropecuária Brasileira, Brasília, v. 45, n. 1, p. 25-31, 2010.

REBOLLAR-ALVITER, A.; MADDEN, L. V.; ELLIS, M. A. Pre- and post-infection activity of azoxystrobin, pyraclostrobin, mefenoxam, and phosphite against leather rot of strawberry, caused by Phytophthora cactorum. Plant Disease, St. Paul, v. 91, n. 5, p. 559-564, 2007.
REUVENI, M. Post-infection applications of $\mathrm{K}_{3} \mathrm{PO}_{3}$, phosphorous acid and dimethomorph inhibit development ofdowny mildew caused by Plasmopara viticola on grapevines. Journal of Small Fruit \& Viticulture, Binghamton, v. 5, n. 22, p. 27-38, 1997.

SANTOS, H. A. A.; DALLA PRIA, M.; SILVA, O. C.; MAY DE MIO, L. L. Controle de doenças do trigo com fosfitos e acibenzolar-s-metil isoladamente ou associados a piraclostrobina + epoxiconazole. Semina: Ciências Agrárias, Londrina, v. 32, n. 2, p. 433-442, 2011.

SILVA, O. C.; SANTOS, H. A. A.; DALLA PRIA, M.; MAY-DE MIO, L. L. Potassium phosphite for control downy mildew of soybean. Crop Protection, Guildford, v. 30, n. 6, p. 598-604, 2011.

SMILLIE, R.; GRANT, B. R.; GUEST, D. The mode of action of phosphite: evidence for both direct and indirect modes of action on three Phytophthora spp. in plants. Phytopathology, Saint Paul, v. 79, n. 9, p. 921-926, 1989.

SÔNEGO, O. R.; GARRIDO, L. R.; CZERMAINSKI, A. B. C. Avaliação da eficácia de algumas marcas comerciais de fosfito de potássio e de fosfonato de potássio no controle do míldio da videira. Bento Gonçalves: Embrapa Uva e Vinho, 2005. 16 p. (Embrapa Uva e Vinho. Circular técnica, 60).

TESSMANN, D. J.; DIANESE, J. C.; GENTA, W.; VIDA, J. B.; MIO, L. L. M. Grape Rust caused by Phakopsora euvitis, a new disease for Brazil. Fitopatologia Brasileira, Brasília, v. 29, n. 3, p. 338, 2004.

TESSMANN, D. J.; VIDA, J. B.; GENTA, W.; KISHINO, A. Y. Doenças e seu manejo. In: KISHINO, A.; CARVALHO, S. L. C. (Ed.). Viticultura tropical o sistema de produção do Paraná. Londrina: Instituto Agronômico do Paraná, 2007. p. 255-293.

VIDA, J. B.; TESSMANN, D. J. Perdas causadas pela ferrugem da videira (Phakopsora euvitis) na cultura da videira. Fitopatologia Brasileira, Brasília, v. 30, p. S140, 2005. Suplemento. 
